\title{
Notification about seasonal influenza vaccination: what is the best way to increase uptake?
}

\author{
See linked article by Van Rossem et al. on pg 308
}

\author{
*Fiona Mowbray, \\ a King's College London, Institute of Psychiatry, London, UK \\ b Emergency Response Department, Health Protection Agency, UK
}

${ }^{*}$ Correspondence: Dr Fiona Mowbray, Institute of Psychiatry, King's College London, Department of Psychological Medicine (PO62), Weston Education Centre, Cutcombe Road, London SE5 9RJ, UK. Tel: +44 (0)1980616968 Fax: +44 (0)1980 612968 E-mail: fiona.mowbray@kcl.ac.uk

Each year, countries undertake public health campaigns to encourage their population to have the seasonal influenza vaccine in an effort to reduce the number of people who will suffer from influenza and influenza-related complications. Influenza vaccines are generally considered to be safe, and are effective in reducing hospital admissions, complications and mortality in at-risk patients.' Ensuring that patients are aware of the availability of the vaccine and are able to make an informed choice about whether to be vaccinated is an important responsibility for primary care physicians. Although the generally poor uptake of seasonal influenza vaccination is well-recognised around the world, ${ }^{1,2}$ the issue is particularly topical now given the disappointing uptake of vaccines during the recent H1N1 influenza pandemic. ${ }^{3}$

In this issue of the $P C R J$, Van Rossem et al. ${ }^{4}$ report findings of an investigation into whether patients of family practices in Belgium want to be notified about the availability of the influenza vaccine. Approximately $80 \%$ of the total study population reported wanting to be notified, a fact which Van Rossem et al. ${ }^{4}$ rightly note should encourage us to be proactive in inviting patients to be vaccinated. Yet around $20 \%$ of patients did not wish to be notified. This figure increased to $34 \%$ among those who had never previously been vaccinated. The authors suggest that this reflects the fact that "some people have already made up their mind about their refusal to be vaccinated" implying that "subjects who had never been vaccinated" had "less need [sic] for notification."

But are we right to assume that such patients have truly exercised informed choice, or do these patients actually have more, not less, need for information and advice? Studies investigating the reasons why some eligible patients do not receive the vaccine have identified a number of important risk factors, many of which do not sit well with our scientific understanding of the risks and benefits of the vaccine. These reasons range from lack of awareness that the vaccine is available and confusion as to who qualifies to receive it, ${ }^{5,6}$ through to perceptions that influenza is not a particularly serious illness, that the vaccine causes influenza, or that the vaccine is likely to cause longterm health effects.? Regardless of personal desires about influenza vaccination notification, it remains the responsibility of health care professionals to ensure that members of the public - particularly those who fall into a risk category - are provided with adequate information about the availability of the vaccine and about the true risks and benefits involved in having it. A proactive notification strategy, as advocated by Van Rossem et al. ${ }^{4}$, is to be applauded - though notification combined with information that specifically targets those misunderstandings or barriers that prevent people from receiving the vaccination may be even better.

Targeting particular vaccination misunderstandings can be a difficult task, and recent research shows that simplistic attempts to correct misconceptions via leaflets could potentially do more harm than good. ${ }^{8}$ Fortunately, Van Rossem et al. ${ }^{4}$ have also highlighted a better way of delivering this information by demonstrating the important role that the family physician plays with regards to the uptake of influenza vaccination. ${ }^{4}$ Among those who wanted to be notified of vaccination opportunities in their study, $85 \%$ reported that they wanted to be notified by their family physician, mostly during a regular consultation. Preference for this is supported by research that shows a significant increase in levels of vaccination following a personal invitation or recommendation from a family physician. ${ }^{5.9}$ But while a face-to-face discussion with a trusted healthcare worker may be the best way of encouraging patients to accept vaccination, we must be mindful that healthcare workers themselves are notoriously bad when it comes to having their seasonal flu vaccination. Within the UK, uptake of the vaccine by healthcare workers languishes at about $37 \%{ }^{10}$ A study by our team investigated the reasons given by healthcare workers for not having the pandemic or seasonal influenza vaccine and found that they were more likely than the general public to cite a belief that the vaccine is dangerous, has side effects or is ineffective. Relying on healthcare workers to communicate about the benefits of a vaccination that they themselves are unwilling to have may be less effective than we might hope.

The study by Van Rossem et al. ${ }^{4}$ has contributed valuable information about the popularity of influenza vaccine notification and preferred notification methods. Evidence-based methods for increasing the impact of this notification should now be a priority. Based on this and existing research it appears that there is a need to produce and disseminate clearer messages to both the general public and healthcare workers about the benefits of the vaccine, the risk of side effects, and who is eligible to be vaccinated.

Conflicts of interest The authors declare that they have no conflicts of interest in relation to this article.

Contributorship The authors both contributed equally to the manuscript. Funding Fiona Mowbray has received research funding from King's College London and the Health Protection Agency, U.K. The views expressed in this publication are those of the authors and are not necessarily those of their funders or employers.

Commissioned article; not externally peer-reviewed; accepted 12th June 2012; online 26th July 2012

C 2012 Primary Care Respiratory Society UK. All rights reserved

http://dx.doi.org/10.4104/pcri.2012.00063

Prim Care Respir J 2012; 21(3): 248-249

\section{References}

1. Nichol KL, Treanor JJ. Vaccines for seasonal and pandemic influenza. J Infect Dis 2006;194:S111-18. http://dx.doi.org/10.1086/507544 
2. Blank PR, Schwenkglenks M, Szucs TD. Influenza vaccination coverage rates in five European countries during season 2006/07 and trends over six consecutive seasons. BMC Public Health 2008;8:272. http://dx. doi.org/10.1186/1471-2458-8-272

3. Brien S, Kwong JC, Buckeridge DL. The determinants of 2009 pandemic A/H1N1 influenza vaccination: a systematic review. Vaccine 2012;30:1255-64. http://dx.doi.org/10.1016/j.vaccine.2011.12.089

4. Van Rossem I, Vandevoorde J, Buyl R, Deridder S, Devroey D. Notification about influenza vaccination in Belgium: a descriptive study of how people want to be informed. Prim Care Respir J 2012;21:308-12. http://dx.doi.org/10.4104/ pcrj.2012.000

5. Kroneman M, van Essen GA, Paget WJ. Influenza vaccination coverage and reasons to refrain among high-risk persons in four European countries. Vaccine 2006; 24:622-8. http://dx.doi.org/10.1016/j.vaccine.2005.08.040

6. Evans MR, Watson PA. Why do older people not get immunised against influenza?
A community survey. Vaccine 2003;21(19-20):2421-7. http://dx.doi.org/10.1016/ S0264-410X(03)00059-8

7. Rubin GJ, Potts HWW, Michie S. Likely uptake of swine and seasonal flu vaccines among healthcare workers. A cross-sectional analysis of UK telephone survey data. Vaccine 2011;29:2421-8. http://dx.doi.org/10.1016/j.vaccine.2011.01.035

8. Skurnik I, Yoon C, Schwarz N. "Myths \& Facts" about the flu: Health education campaigns can reduce vaccination intentions. 2007. h ttp://www. granthalliburton.org/images/Skurnik.pdf

9. Lewis-Parmar H, McCann R. Achieving national influenza vaccine targets - an investigation of the factors affecting influenza vaccine uptake in older persons and people with diabetes. Commun Dis Public Health 2002;5:119-26.

10. O'Reilly FW, Cran GW, Stevens AB. Factors affecting influenza vaccine uptake among health care workers. Occup Med 2005;55:474-9.

http://dx.doi.org/10.1093/ occmed/kqi099

\section{Understanding the true burden of COPD: the epidemiological challenges}

See linked article by McKay et al. on pg 313

\section{*Sundeep S Salvi ${ }^{\mathrm{a}}$, Roslina Manap ${ }^{\mathrm{b}}$, Richard Beasley}

a Chest Research Foundation, Pune, India,

b Universiti Kebangsaan Malaysia Medical Centre, Kuala Lumpur, Malaysia,

Medical Research Institute of New Zealand, Wellington, New Zealand

*Correspondence: Dr Sundeep S Salvi, Director, Chest Research Foundation, Marigold Complex, Kalyaninagar, Pune 411014, India Tel: +919921211000 Fax: +912027035371

E-mail: ssalvi@crfindia.com

According to the World Health Organization (WHO), an estimated 3 million people die due to chronic obstructive pulmonary disease (COPD) every year, making it the fourth leading cause of death in the world. ${ }^{1}$ Around $90 \%$ of these deaths occur in low- and middleincome countries, with India and China alone estimated to account for $66 \%$ of total global COPD mortality. ${ }^{2}$ By 2020 , it is anticipated that COPD will become the third leading cause of death in the world. ${ }^{1}$ The largest number of deaths will be in the South East Asian region, where mortality due to COPD is expected to grow by $160 \%$, totalling more than the combined numbers of deaths due to malaria, tuberculosis and HIV/AIDS.

However, these data may well underestimate the problem. Tan et al. ${ }^{4}$ estimated COPD prevalence in the Asia Pacific region to be $6.3 \%$, compared to the rate of $3.9 \%$ extrapolated from $\mathrm{WHO}$ data. Similarly, COPD prevalence in China was found to be 2.5 times greater $^{5}$ than that estimated by WHO. Despite this apparently huge burden, there are few good quality epidemiological studies on COPD prevalence in South East Asia. For several decades the major health burden has been communicable diseases such as tuberculosis, malaria and HIVIAIDS; governments, lay people and physicians have been slow to recognise the impact of COPD. Yet with the increasing morbidity and mortality from COPD - which has already overtaken most communicable diseases as a leading cause of death - there is now a need for more intensive research and analysis of the epidemiology of COPD in this region.

In this issue of the $P C R J$, McKay and colleagues report a systematic review on the prevalence of COPD in India. ${ }^{6}$ India is one of the largest countries in Asia with an estimated population of over 1.2 billion, where small increases in the percentage prevalence of a disease can translate into large increases in the number of cases. This huge population is exposed to a large number of COPD risk factors. Tobacco smoking rates in India vary from $13.3 \%$ to $59.5 \%$ amongst men and $0.2 \%$ to $22 \%$ amongst women. ${ }^{7}$ Around $70 \%$ of smokers in India smoke 'bidis' instead of cigarettes, which contain crude tobacco (0.15-0.25g) loosely packed in hand-rolled dried leaves of tendu (Diisopyros melanoxylon). The smoke from bidis contains 5 times more tar than the smoke from cigarettes, making bidi smoking a far greater risk factor for COPD than cigarette smoking. ${ }^{8}$ But more importantly, over $70 \%$ of homes in India use biomass fuel for cooking and heating, which again poses a greater risk factor for COPD than tobacco smoking. ${ }^{9}$ The prevalence of other risk factors such as early childhood lower respiratory tract infections, post-pulmonary tuberculosis, chronic poorly controlled asthma, poor socioeconomic status and a growing ageing population is also very high.

McKay et al. reviewed a total of 16 papers on COPD prevalence published from 1980 onwards. Disappointingly, they could not identify a single study that provided a rigorous estimate of COPD prevalence using a relatively standard spirometry-based definition, and were therefore unable to perform a meta-analysis. ${ }^{6}$ Five of the 16 studies examined the prevalence of chronic bronchitis as a surrogate for COPD using validated questionnaires, while the remaining studies defined COPD using less traditional and non-standard methods. The results of this study beg some important questions:

- What are the standard research tools and methodology needed to define COPD in population studies?

- And why has a country like India not been able to conduct such a study?

Unlike asthma (a term coined by Hippocrates over 3000 years ago), COPD is a relatively new disease that has undergone remarkable changes in its terminology over the last 350 years. In the early 1800s, the terms 'bronchitis' (introduced by the British physician Charles Badham) and 'emphysema' (introduced by the Frenchman Laennec) were proposed. For the next 100 years 'emphysema' was used largely by American physicians, and 'chronic bronchitis' by the British, and in 